\title{
Aspect Ratio Control of Layered Double Hydroxide Nanosheets and their Application for High Oxygen Barrier Coating in Flexible Food Pack- aging
}

\author{
Jingfang Yu, Jean-Charles Buffet, Dermot O'Hare*
}

Chemistry Research Laboratory, Department of Chemistry, University of Oxford, 12 Mansfield Road, Oxford, OX1 3TA, UK.

\begin{abstract}
We report a method to rationally control of the aspect ratio of layered double hydroxide for use in a barrier coating for food packaging films. The reconstruction of a $\mathrm{Mg}_{2} \mathrm{Al}$-layered double oxide (LDO) in concentrated aqueous glycine solutions produces dispersions of $\mathrm{Mg}_{2} \mathrm{Al}-\mathrm{LDH}$ nanosheets. The nanosheet thickness decreases and diameter increases with increasing reconstruction time from 16 to $96 \mathrm{~h}$. We observe a limiting nanosheet aspect ratio of ca. $336 \pm 170$. These $\mathrm{Mg}_{2} \mathrm{Al}-\mathrm{LDH}$ nanosheets can be dispersed in PVA to give a water-based dispersion that can be used to coat flexible polymeric films. Oxygen transmission rate (OTR) of a PET film decreases when the thickness of the dried coated layer increases, an OTR of $<0.005 \mathrm{~mL} \cdot \mathrm{m}^{-2} \cdot$ day $^{-1}$ is observed for a coating with thickness of $1175 \pm 101 \mathrm{~nm}$.
\end{abstract}

\section{INTRODUCTION}

Non-recyclable food packaging film is becoming a major global concern to human life as they can take decades to decompose in natural environment. In particular, there is an urgent unmet demand to find a replacement for aluminum metallization within the film structure and films containing aluminum foil that create the necessary oxygen and moisture barrier performance. Currently, it is uneconomic to separate metallized layers from a polymer film and as a result, metallized food packaging films cannot enter the plastic circular economy.

Currently, food packaging industry typically requires an oxygen transmission rate on the order of $10^{-1} \mathrm{~mL} \cdot \mathrm{m}^{-2} \cdot$ day $^{-1}$. Both theoretical calculations ${ }^{1}$ and recent experiment work ${ }^{2}$, 3 have identified high aspect ratio inorganic platelets as one of the most promising approaches to improve the oxygen barrier of polymeric films. Assuming a "block tiled" structure, then a torturous path model, the platelet aspect ratio (diameter divided by thickness) is one of the most important factors for building a high oxygen/moisture barrier layer. Furthermore, if such platelets are to be used to create a barrier layer in food related packaging then non-toxic chemistry needs to be used throughout.

Previously, inorganic layered materials such as MMT,3-5 graphene, ${ }^{6-8}$ and $\alpha$-zirconium phosphate ${ }^{9}$, to have been shown to improve the gas barrier properties of a polymeric films, the current state-of-the-art approach involves a layer-by-layer assembly ${ }^{11}$ of impermeable platelets and a binder. However, this approach would not be practicable solution for large scale, high speed barrier film production. Layered double hydroxides (LDHs) have also shown to offer promising barrier opportunities, ${ }^{12-14}$ as such they constitute a large family of synthetic layered materials that are commonly represented by the formula, $\left[\mathrm{M}_{1-}\right.$ $\left.{ }_{x} \mathrm{M}_{x}{ }_{x}(\mathrm{OH})_{2}\right]^{a+}\left[\mathrm{A}^{n-}{ }_{a / n}\right] \cdot m \mathrm{H}_{2} \mathrm{O}$ ( $\mathrm{M}$ and $\mathrm{M}^{\prime}$ are most commonly divalent and trivalent cations respectively, $\mathrm{A}^{n-}$ is the interlayer anion, and $\mathrm{o}<x<1)$. Hydrotalcites, $\left[\mathrm{Mg}_{1-}\right.$ $\left.{ }_{x} \mathrm{Al}_{x}(\mathrm{OH})_{2}\right]\left(\mathrm{CO}_{3}\right)_{2} / x^{\bullet} m \mathrm{H}_{2} \mathrm{O},\left(\mathrm{Mg}_{y} \mathrm{Al}-\mathrm{CO}_{3}\right.$-LDHs; $\left.\left.y=1-x / x\right)\right)$ can be fully synthetic and have been recognized as food contact safe materials. ${ }^{15}$

Conventionally prepared $\mathrm{Mg}_{y} \mathrm{Al}-\mathrm{LDHs}$ exhibit low aspect ratios, exfoliation provides a route to thin nanosheets that improves the aspect ratios of as-prepared platelets,$^{16}$ however this process requires a high dielectric constant medium such as formamide which is highly toxic. ${ }^{16,17}$ Bottomup processes have also been successfull, ${ }^{18}$ but the nanosheet yield is low and the synthesis conditions generate large amounts of by products and waste solvent. We recently reported a reconstruction method to synthesize high aspect ratio, food safe $\mathrm{Mg}_{2} \mathrm{Al}-\mathrm{LDH}$ nanosheets (NS) using nontoxic chemistry. ${ }^{19} \mathrm{Mg}_{2} \mathrm{Al}-\mathrm{CO}_{3} \mathrm{LDH}$ was first calcined at $45^{\circ}$ ${ }^{\circ} \mathrm{C}$ to form a layered double oxide (LDO), then reconstruction into high aspect ratio $\mathrm{Mg}_{2} \mathrm{Al}-\mathrm{CO}_{3} \mathrm{NS}$ was accomplished using a concentrated aqueous glycine solution. This is a facile method that it does not require a special $\mathrm{Mg}_{\mathrm{x}} \mathrm{Al}-\mathrm{LDH}$ precursor or inert gas protection as compared to previous exfoliation examples. ${ }^{20,21}$

In this report, we focused on the reconstruction of $\mathrm{Mg}_{2} \mathrm{Al}-$ LDO in concentrated aqueous glycine solution. As a result, we have been able to exploit the reconstruction method to prepare a range of $\mathrm{Mg}_{2} \mathrm{Al}-\mathrm{LDH}$ nanosheet samples with different aspect ratio by fine-tuning the calcination temperature and LDO reconstruction time. We find the aspect ratios of reconstructed LDH NS samples is the highest using LDO calcined at $550^{\circ} \mathrm{C}$ in 
Scheme 1. Schematic representation of the anisotropic crystal growth during reconstruction of the LDO, crystal growth inhibition of the LDH is observed along the platelet stacking axis (c-axis) as a function of time and glycine concentration

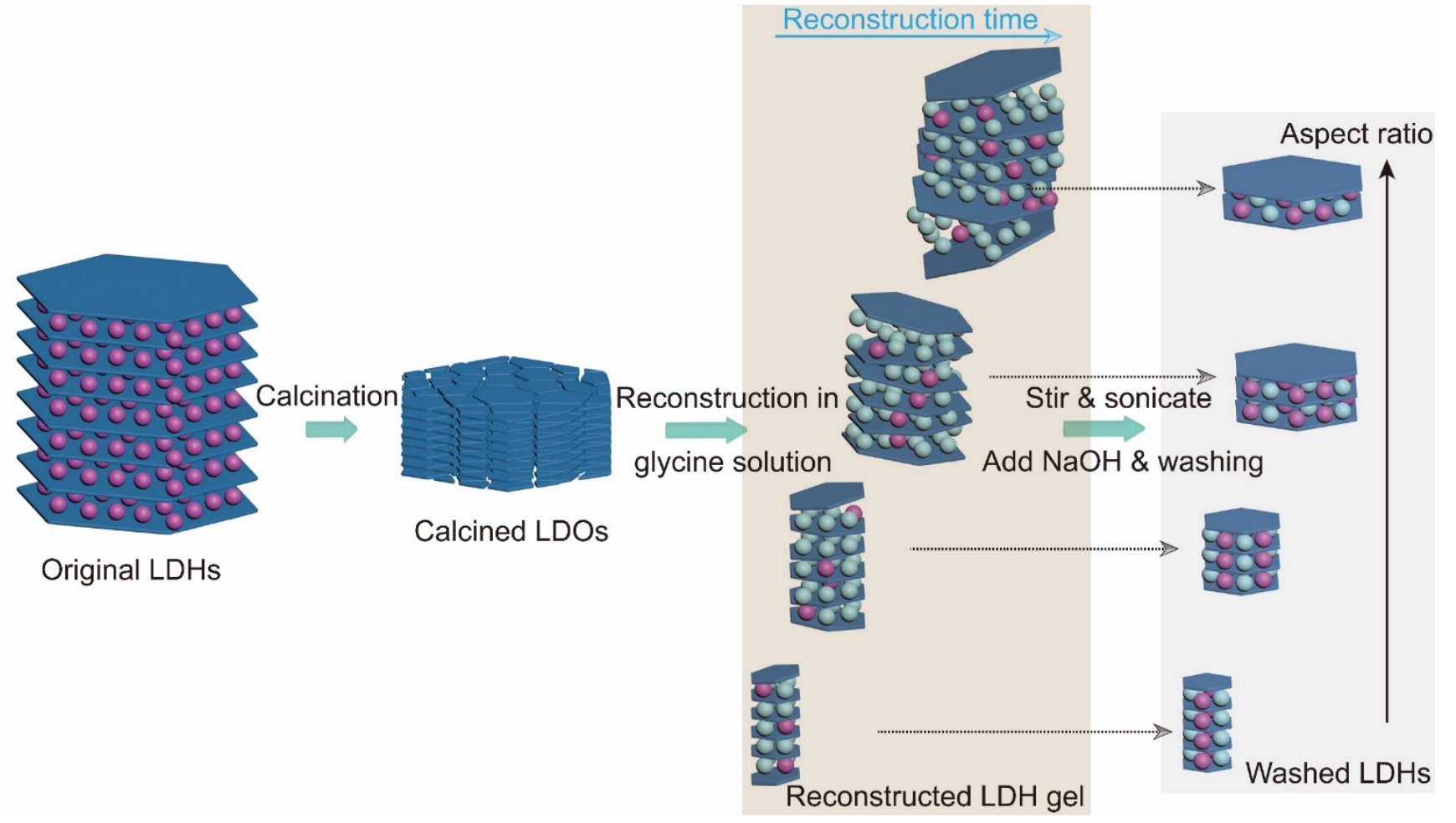

combination with longer reconstruction times (Scheme 1). The obtained $\mathrm{Mg}_{2} \mathrm{Al}-\mathrm{LDH}$ nanosheet samples have aspect ratio ranging from ca. $87 \pm 17$ to $336 \pm 170$. We prepared stable aqueous coating dispersions containing $\mathrm{Mg}_{2} \mathrm{Al}-\mathrm{LDH}$ NS and poly(vinyl alcohol) (PVA) mixture. A $12 \mu \mathrm{m}$ PET film showed significant oxygen barrier improvement after coating. The relative permeability of the LDH/PVA coated layer decreases as the aspect ratio of LDH nanosheets are increased, the experimental data agrees well with theoretical values calculated on the basis of tortuous pathway models. Oxygen transmission rate (OTR) of the LDH/PVA coated PET film decreased significantly when the dried coat layer thickness is increased, reaching below the instrument detection limit $\left(<0.005 \mathrm{~mL} \cdot \mathrm{m}^{-2}\right.$.day $\left.{ }^{-1}\right)$ at a film thickness of $1175 \pm 101 \mathrm{~nm}$. Furthermore, the coated barrier film is mechanically flexible, transparent and suitable for food packaging.

\section{RESULTS AND DISCUSSIONS}

Rational preparation of high aspect ratio LDH nanosheets. The reconstruction of an amorphous LDO back to a crystalline LDH is well known, this process is often referred to as "a memory effect". ${ }^{22,} 23$ For example, during calcination of $\mathrm{Mg}_{2} \mathrm{Al}-\mathrm{CO}_{3} \mathrm{LDH}$ the layered structure collapses by dehydroxylation of the metal hydroxide layers and by interlayer carbonate ion decomposition releasing $\mathrm{CO}_{2}$, upon rehydration in air the crystalline layered structure reforms with concomitant $\mathrm{CO}_{2}$ capture from the atmosphere to form the interlayer charge compensating carbonate anions. Our earlier work indicates that reconstruction is actually a dissolution-recrystallisation ${ }^{24}, 25$ process and that high aspect ratio LDH NS can be prepared using an aqueous concentrated glycine solution. ${ }^{19}$ Therefore, in order to gain more insight into this process we have explored if the aspect ratio of the isolated LDH nanosheets can be rationally controlled by the key process parameters such as calcination temperature, dielectric constant of the reconstruction solution and duration of reconstruction.

Platelet samples of $\left[\mathrm{Mg}_{0.66} \mathrm{Al}_{0.33}(\mathrm{OH})_{2}\right]\left(\mathrm{CO}_{3}\right)_{0.17} \cdot m \mathrm{H}_{2} \mathrm{O}$; $\left(\mathrm{Mg}_{2} \mathrm{Al}-\mathrm{CO}_{3} \mathrm{LDH}\right.$ ) with diameter ca. $7 \mu \mathrm{m}$ (Figure $\mathrm{S} 1$ ) were first calcined at 450,550 , or $750{ }^{\circ} \mathrm{C}$ for $12 \mathrm{~h}$ to obtain the corresponding LDOs (Figure Sia and Figure S2). The obtained LDOs were then reconstructed in $2 \mathrm{M}$ glycine solution at $100{ }^{\circ} \mathrm{C}$ to restore the original $\mathrm{LDH}$ structure (Scheme 1). Surprisingly, we found that the aspect ratio of the obtained LDH NS samples increased with increasing reconstruction time from 16 to $96 \mathrm{~h}$ (Figure 1a-d and g), reaching a limiting nanosheet aspect ratio of ca. $336 \pm 170$ when the $550^{\circ} \mathrm{C}$ calcined LDO sample was reconstructed for $96 \mathrm{~h}$. The AFM data show the reconstructed LDH particles are thin nanosheets (Figure $\mathrm{S}_{3}$ and Table $\mathrm{S} 1$ ), we clearly observe the nanosheet thickness decreases (Figure $\mathrm{S}_{4} \mathrm{a}$ ) and diameter increases (Figure $\mathrm{S}_{4} \mathrm{~b}$ ) with reconstruction time. The LDH NS formed from an LDO prepared at $450^{\circ} \mathrm{C}$ have an average platelet diameter ca. $200 \mathrm{~nm}$ (Figure $\mathrm{S}_{5} \mathrm{a}$ ) which is a remarkable reduction from the $7 \mu \mathrm{m}$ diameter of the parent LDO. Previous study found that uptake of amino acids increases with reconstruction time. ${ }^{26}$ As the concentration of glycine in the confined interlayer region increases with time, we propose the dielectric constant in the region surrounding the platelets also increases with reaction time. After the reconstruction 


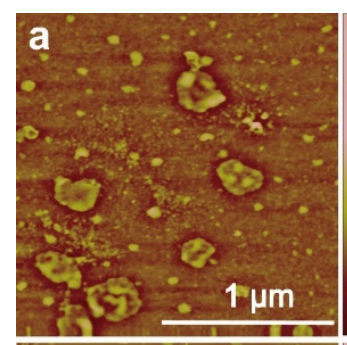

$.7 .5 \mathrm{~nm}$
$.5 \mathrm{~nm}$
$2.5 \mathrm{~nm}$
0
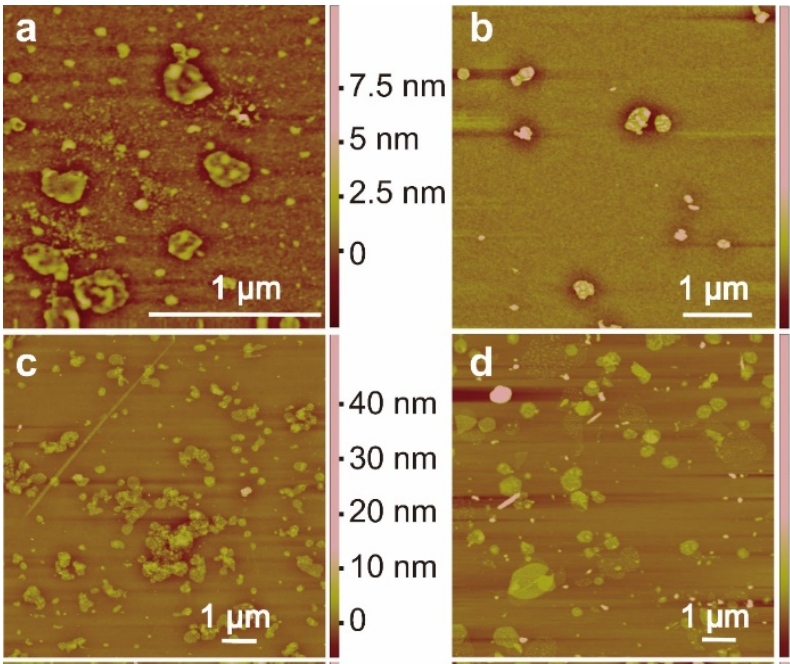

$\cdot 16 \mathrm{~nm}$

$\cdot 12 \mathrm{~nm}$

$.8 \mathrm{~nm}$
$.4 \mathrm{~nm}$
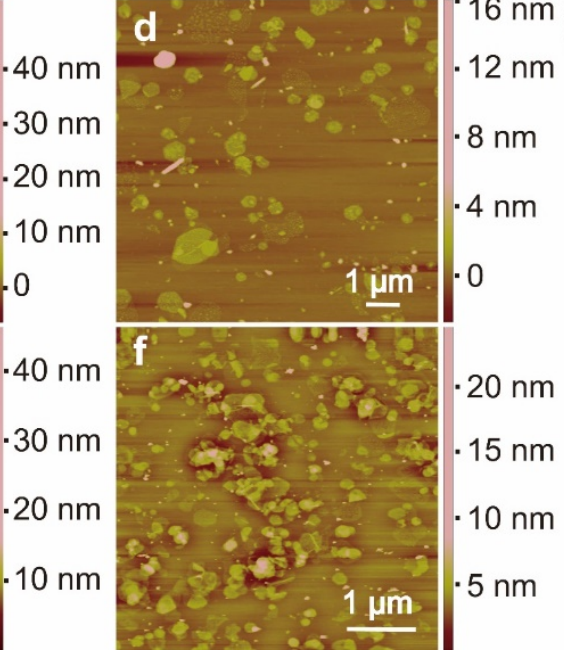

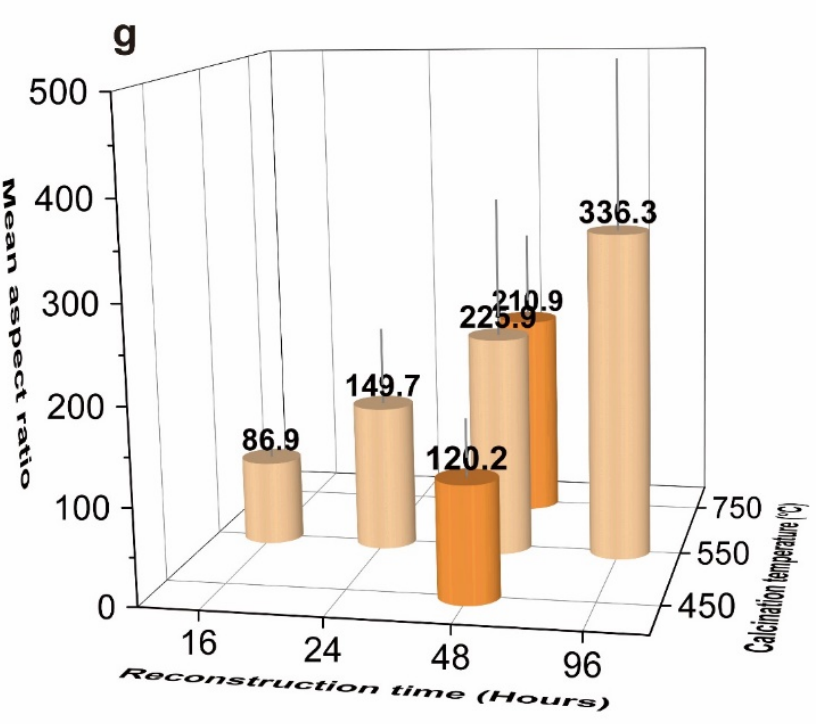

Figure 1. AFM measurements of the reconstructed LDH nanosheets. AFM images of LDOs calcined at $550^{\circ} \mathrm{C}$ followed by reconstruction in glycine solution for: a, 16 h, b, 24 h, c. 48 h, d, 96 h. AFM images of LDO calcined at: e, $450^{\circ} \mathrm{C}$ and f, $750{ }^{\circ} \mathrm{C}$ followed by reconstruction time in glycine for $48 \mathrm{~h}$. g, $3 \mathrm{D}$ correlation of platelet aspect ratio as a function of LDO reconstruction time and calcination temperature.

reaction, a semi-transparent $\mathrm{LDH}$ gel is formed, this gellike phase can be re-dispersed in water by stirring overnight and sonication for 5 minutes (Scheme 1).

We postulate that a large local dielectric constant within the interlayer region weakens the interlayer electrostatic interactions leading to a loosely attracted lamellar structure (Scheme 1). Thus, the LDH gel sample prepared from the longest reconstruction period is the most susceptible to shear stresses. During stirring and post reconstruction sonication, the shear force provides enough energy to exfoliate this loosely attached lamellar structure (Scheme 1) ${ }^{16}$ We find that the aspect ratio of the LDH NS reconstructed for $16 \mathrm{~h}$ is ca. $87 \pm 17$ (Figure 1a and g), ca. twice larger than that of the original LDH $(38 \pm 14)$ (Figure S6). When a longer reconstruction time $(24 \mathrm{~h})$ was used, the aspect ratio of the LDH NS increased to ca. $150 \pm 80$ (Figure $1 \mathrm{~b}$ and $\mathrm{g}$ ). The aspect ratio is $226 \pm 140$ (Figure 1c and g) when the reconstruction time was extended to $48 \mathrm{~h}$ and reaches the highest value of $336 \pm 170$ (Figure 1d and g) using a $96 \mathrm{~h}$ reconstruction time.

The effect of calcination temperature on the aspect ratio of obtained LDH samples was investigated. In addition to $55^{\circ}$ ${ }^{\circ} \mathrm{C}$, the original LDH was calcined at 450 as well as $750^{\circ} \mathrm{C}$ for $12 \mathrm{~h}$ to prepare LDOs. These two LDO samples (450 and $750{ }^{\circ} \mathrm{C}$ calcined) were used for reconstruction under the same condition as that of the $550^{\circ} \mathrm{C}$ calcined LDO in glycine solution for $48 \mathrm{~h}$ (Figure $\mathrm{S}_{5} \mathrm{a}$ and $\mathrm{b}$ ). The reconstructed LDH nanosheet samples have aspect ratio of ca. $120 \pm 58$ (from $450{ }^{\circ} \mathrm{C}$ calcined LDO) (Figure 1e and g), 226 \pm 140 (from $550^{\circ} \mathrm{C}$ calcined LDO) (Figure $1 \mathrm{c}$ and g), and 211 \pm 92 (from $750{ }^{\circ} \mathrm{C}$ calcined LDO) (Figure if and g). The aspect ratio of reconstructed $\mathrm{LDH}$ samples increased and then decreased with the calcination temperature and the maximum value reached at the LDH sample prepared from $550{ }^{\circ} \mathrm{C}$ calcined LDO (Figure 1g). The surface area and pore volume of the original LDH increased when calcined to obtain LDO (Figure $\mathrm{S}_{7}$ and table $\mathrm{S}_{2}$ ). The surface area increased from 4 to $130 \mathrm{~m}^{2} / \mathrm{g}$ upon calcination at $450{ }^{\circ} \mathrm{C}$ for $12 \mathrm{~h}$. The surface area of the LDO increased to $202 \mathrm{~m}^{2} / \mathrm{g}$ when calcined at $550{ }^{\circ} \mathrm{C}$. Upon further heating to $750{ }^{\circ} \mathrm{C}$ the surface area of the LDO decreases to $125 \mathrm{~m}^{2} / \mathrm{g}$, this type of behavior has been seen elsewhere and is associated of sintering and onset of dense spinel. ${ }^{24,}{ }^{27}$ A large surface area and pore volume increase the chance of glycine absorption during the reconstruction step. Utilizing a LDO sample with higher surface area and pore volume increases glycine uptake and thus increases the local glycine concentration giving a higher dielectric constant in the interlayer region. Similar to the reconstruction time effect, a high dielectric constant in the interlayer area weakens the electrostatic interactions and better facilitates exfoliation. Based on TGA results (Figure S8), the glycine content is the highest when $550{ }^{\circ} \mathrm{C}$ calcined LDO was used during the reconstruction step. The LDH NS contain ca. 16, 19, and $12 \mathrm{wt} \%$ glycine when 450 , 550 and $750{ }^{\circ} \mathrm{C}$ calcined LDO was used (Table $\mathrm{S}_{3}$, Supporting Information).

In concentrated glycine solution we postulate that crystal growth inhibition along the stacking axis (c-axis) results in conversion of original large platelet (low aspect ratio) 


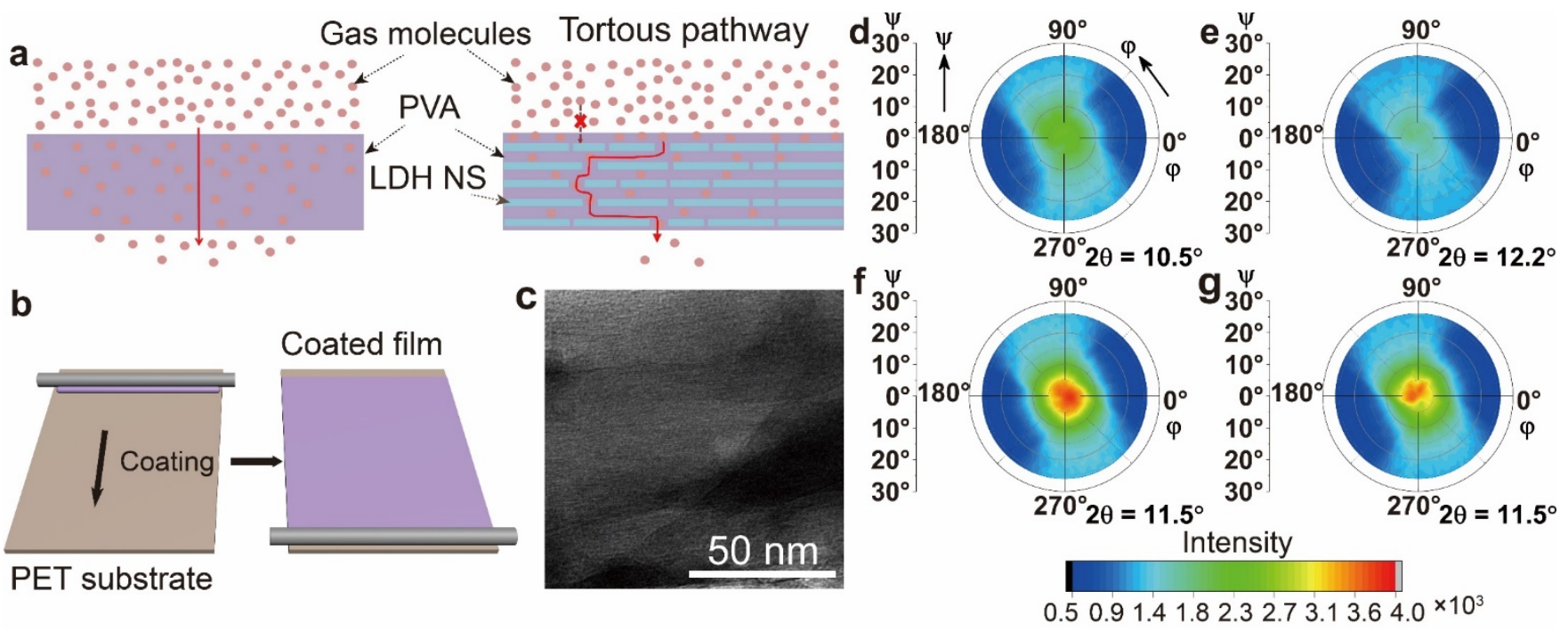

Figure 2. Coated film preparation and film structure characterizations. a) Tortuous pathway assembled by the aligned LDH NS in PVA matrix providing longer diffusion pathway for gas molecules compared to a pure PVA layer. b) Bar coating process. c) Cross-sectional TEM showing the well-aligned LDH NS $\left(55^{\circ}{ }^{\circ} \mathrm{C}-48 \mathrm{~h}\right)$ in the coated layer. Pole figure measurements of LDH/PVA phase and $\mathrm{LDH} /$ Glycine phase in the barrier films containing $\mathrm{LDH}$ samples $\left(55^{\circ}{ }^{\circ} \mathrm{C}\right)$ with different aspect ratio values of $87(16 \mathrm{~h})$ ( $\mathrm{d}$ and e), $150(24 \mathrm{~h})(\mathrm{f})$, and $336(96 \mathrm{~h})(\mathrm{g})$ in the coated layer. All the LDH/PVA coated films discussed in this figure were prepared from a $5 \mathrm{wt} \%$ coating solution ( $3 \mathrm{wt} \% \mathrm{LDH}$ and $2 \mathrm{wt} \% \mathrm{PVA}$ ), the coating gap was $24 \mu \mathrm{m}$.

LDH sample towards high aspect ratio LDH NS, we ascribe this effect is due to the high dielectric constant of concentrated glycine solution. The dielectric constant of the solution scales with amino acid concentration. ${ }^{28}$ The inhibition effect scales with the amount of glycine molecules absorbed in the interlayer region and on the layer surface.

Preparation of LDH Nanosheet/PVA coated PET films. Solid samples of LDH nanosheets may be recovered from water (Scheme 1) by precipitation upon addition of $\mathrm{NaOH}$. The precipitated LDH nanosheets were centrifuged, washed with water to remove the excess glycine and $\mathrm{NaOH}$. Solid LDH nanosheet samples can be mixed with a $10 \mathrm{wt} \%$ PVA $\left(\mathrm{M}_{\mathrm{W}} 67, \mathrm{ooo} \mathrm{g} \cdot \mathrm{mol}^{-1}\right)$ stock solution to prepare coating solutions. The LDH sample used to prepare these coating dispersions are referred to as LDH (X-Y), where X is the calcination temperature and $\mathrm{Y}$ is the reconstruction time in glycine solution. For example, $\mathrm{LDH}\left(55^{\circ}{ }^{\circ} \mathrm{C}-16 \mathrm{~h}\right)$ refers to the LDH NS sample prepared from an LDO prepared at $550{ }^{\circ} \mathrm{C}$ followed by reconstruction in glycine solution for $16 \mathrm{~h}$. All the LDH/PVA coating solution samples have an LDH:PVA weight ratio of 6o:40, corresponding to $48 \mathrm{vol} \% \mathrm{LDH}$ in the dried coat layer (see Supporting Information). Coating solutions are referred to their total solid content. For example, a $5 \mathrm{wt} \% \mathrm{LDH} / \mathrm{PVA}$ coating solution contains $3 \mathrm{wt} \% \mathrm{LDH}$ and $2 \mathrm{wt} \%$ PVA where water is 95 wt $\%$.

In an idealized model, ${ }^{1}$ assuming a polymeric barrier membrane contains impermeable platelets aligned periodically parallel to the plane of the film, diffusion occurs through the space between the platelets. The extra diffusion pathway (tortuous pathway), the solute molecule has to travel across the film significantly decreases the diffusion rate compared to that of a pure polymeric film (Figure 2a). The reduction of permeability is proportional to the aspect ra- tio of the impermeable platelets, the higher the platelet aspect ratio the greater the reduction of permeability. ${ }^{2}$ The alignment of the impermeable platelets relative to the film surface is also another important factor. The tortuosity is the highest when the platelets are perfectly aligned perpendicular to the diffusion pathway. The higher the degree of alignment the longer the diffusion pathway. Thus, the reduction of permeability is the highest when a polymeric matrix contains a high aspect ratio platelets with the platelets parallel aligned perpendicular to the diffusion pathway.

We have applied the LDH/PVA coating solutions on PET film substrate using a bar coater (Figure $2 \mathrm{~b}$ ) in a single coating step. The cross-sectional TEM image (Figure 2c) shows that the LDH NS in the coated layer well aligned within the PVA matrix. Further structural studies have been performed using X-ray powder diffraction (Figure S9), the XRD shows a broad feature $\left(2 \theta\right.$ ca. $\left.8^{\circ}-14^{\circ}\right)$ for coated film samples containing reconstructed $\mathrm{LDH}$ nanosheets. Two Bragg reflections at $2 \theta=10.5^{\circ}$ and $11.5^{\circ}$ corresponding to LDH/PVA and LDH/glycine phase intercalation phases with interlayer separations of $8.4 \AA$ and 7.7 $\AA$ respectively (Figure S9). When an LDH sample $\left(55^{\circ}{ }^{\circ} \mathrm{C}\right.$ $16 \mathrm{~h})$ with low aspect ratio $(87 \pm 17)$ was used, the diffraction peak of the coated film sample relating to LDH/PVA phase was stronger than the diffraction peak from $\mathrm{LDH} /$ glycine phase. When LDH samples $\left(55^{\circ}{ }^{\circ} \mathrm{C}-24 \mathrm{~h}\right.$ and $96 \mathrm{~h}$ ) with higher aspect ratio (150 \pm 80 and $336 \pm 170$ ) were used, the peak center of the coated film samples shifted to $\mathrm{LDH} /$ glycine phase. This is related to the degree of alignment of LDH in the coated layer. Most of the particles aligned to the plane relating to $\mathrm{LDH} /$ glycine phase for the coated film samples containing higher aspect ratio $(150 \pm$ 80 and $336 \pm 170) \mathrm{LDH}$ samples $\left(550{ }^{\circ} \mathrm{C}-24\right.$ and $96 \mathrm{~h}$ ) while relatively more particles aligned to the plane relating to 
LDH/PVA phase for the coated film sample containing low aspect ratio $(87 \pm 17) \mathrm{LDH}$ sample $\left(550^{\circ} \mathrm{C}-16 \mathrm{~h}\right)$.

Pole figure measurements (Figure $2 \mathrm{~d}$-e) were used to accurately determine the degree of alignment of LDH layers as a function of aspect ratio with poles fixed at the planes relating to the LDH/PVA and LDH/glycine phases. A measurement was made with the detector fixed at $2 \theta=11.5^{\circ}$ in for each coated film sample and an addition measurement was made for the sample containing the lowest aspect ratio LDH sample of $87\left(550^{\circ} \mathrm{C}-16 \mathrm{~h}\right)$ with $2 \theta=10.5^{\circ}$. The pole figures display the scattering from $\mathrm{LDH}$ layers obtained by tilting $(\psi)$ and rotating $(\varphi)$ a film sample at an angle away from the surface normal. We averaged the scattering from all rotating angles $(\varphi)$ and plotted the intensity as a function of sample tilting angle $\varphi$ (Figure Sio a-c). In order to compare the oriental distribution, the full width at half maximum height (FWHM) were estimated by fitting the curve with a Gaussian distribution (Equation S1, Supporting Information). The fitted FWHM values (Figure Siod) of the coated films containing reconstructed LDH nanosheet samples with different aspect ratio of ca. $87 \pm 17\left(550^{\circ} \mathrm{C}-16\right.$ h), ca. $150 \pm 80\left(550{ }^{\circ} \mathrm{C}-24 \mathrm{~h}\right)$, and $336 \pm 170\left(550^{\circ} \mathrm{C}-96 \mathrm{~h}\right)$ are $20 \pm 0.3^{\circ}, 17.7 \pm 0.2^{\circ}$, and $17.2 \pm 0.3^{\circ}$ respectively for the $\mathrm{LDH} /$ glycine phase. In addition, the fitted FWHM value of the LDH/PVA phase of the film containing LDH with aspect ratio of ca. $87 \pm 17\left(550^{\circ} \mathrm{C}-16 \mathrm{~h}\right)$ is $20 \pm 0.3^{\circ}$ same as the fitted value of the $\mathrm{LDH} /$ glycine phase. The results indicated that the alignment distribution is wider when a coated film contains LDHs with a lower aspect ratio $(550$ ${ }^{\circ} \mathrm{C}-16 \mathrm{~h}$ ). The degree of orientation (Equation S2, Supporting Information) is calculated to be $88.9 \pm 0.2 \%$ for the coated film containing LDH sample with aspect ratio of ca. $87 \pm 17\left(550^{\circ} \mathrm{C}-16 \mathrm{~h}\right)$ for both phases. It is $90.2 \pm 0.2 \%$ and $90.4 \pm 0.1 \%$ for film samples containing LDH samples with aspect ratio of ca. $150 \pm 80\left(550^{\circ} \mathrm{C}-24 \mathrm{~h}\right)$ and $336 \pm 170(550$ ${ }^{\circ} \mathrm{C}-96 \mathrm{~h}$ ) (Table 1). The oriental distribution of the film sample containing LDH sample $\left(550{ }^{\circ} \mathrm{C}-96 \mathrm{~h}\right)$ with the highest aspect ratio of $336 \pm 170$ is slightly higher than the film sample containing LDH sample $\left(550^{\circ} \mathrm{C}-24 \mathrm{~h}\right)$ of aspect ratio of $150 \pm 80$. The small differences is probably due to the wide aspect ratio distribution of the LDH samples. The overall trend is that the degree of orientation of $\mathrm{LDH}$ nanosheets within the coated film is highest when using nanosheets with the highest aspect ratio.

Table 1. Degree of orientation as a function of LDH aspect ratio. The LDH samples were prepared from LDO prepared at $550^{\circ} \mathrm{C}$. All the coated films discussed in this table were prepared from a $5 \mathrm{wt} \%$ coating solution ( $3 w t \%$ LDH and $2 \mathrm{wt} \%$ PVA), the coating gap was 24 $\mu \mathrm{m}$.

\begin{tabular}{ll}
\hline Aspect ratio & $\begin{array}{l}\text { Degree of Ori- } \\
\text { entation }(\%)\end{array}$ \\
\hline $87 \pm 17(16 \mathrm{~h})(\mathrm{LDH} / \mathrm{PVA})$ & $88.9 \pm 0.2$ \\
$87 \pm 17(16 \mathrm{~h})(\mathrm{LDH} /$ glycine $)$ & $88.9 \pm 0.2$ \\
$150 \pm 80(24 \mathrm{~h})(\mathrm{LDH} /$ glycine $)$ & $90.2 \pm 0.2$ \\
\hline
\end{tabular}

$336 \pm 170(96 \mathrm{~h})(\mathrm{LDH} /$ glycine $) \quad 90.4 \pm 0.1$

Oxygen barrier measurements of LDH nanosheet/PVA coated PET films. At $23{ }^{\circ} \mathrm{C}$, and o\% relative humidity, the $12 \mu \mathrm{m}$ PET substrate has an OTR of 133.5 $\mathrm{mL} \cdot \mathrm{m}^{-2}$.day ${ }^{-1}$ corresponding to an $\mathrm{O}_{2}$ permeability of $18.3 \times$ $10^{-16} \mathrm{~cm}^{3} \cdot \mathrm{cm} \cdot \mathrm{cm}^{-2} \cdot \mathrm{s}^{-1} \cdot \mathrm{Pa}^{-1} \cdot 19$ The OTR of the film decreased to $18.25 \mathrm{~mL} \cdot \mathrm{m}^{-2} \cdot \mathrm{day}^{-1}$ when the PET is coated with $5 \mathrm{wt} \%$ PVA solution, giving a dry coated layer thickness of ca. 890 $\pm 32 \mathrm{~nm} .{ }^{19}$ The permeability of the coated layer is obtained based on an ideal laminate theory. ${ }^{29-31}$ When the PET substrate is coated with a coating solution containing $3 \mathrm{wt} \%$ LDH NS and $2 \mathrm{wt} \%$ PVA (total solid content is $5 \mathrm{wt} \%$ ) at a $24 \mu \mathrm{m}$ coating gap (the coating gap is pre-determined, refers to the wet film thickness of coating layer), the permeability of the LDH/PVA coated layer (P) are o.oo12o, $0.00095,0.00095,0.00073,0.00085$, and $0.0007 \times 10^{-16}$ $\mathrm{cm} 3 \cdot \mathrm{cm} \cdot \mathrm{cm}^{-2} \cdot \mathrm{s}^{-1} \cdot \mathrm{Pa}^{-1}$ when the mean aspect ratio of $\mathrm{LDH}$ samples are $87,120,150,211,226$, and 336 respectively (Table $\mathrm{S}_{4}$, Supporting Information). The permeability of the PVA coated layer $\left(\mathrm{P}_{\mathrm{o}}\right)$ is $0.186 \times 10^{-16} \mathrm{~cm} \cdot \mathrm{cm}^{3} \cdot \mathrm{cm}^{-2} \cdot \mathrm{s}^{-1} \cdot \mathrm{Pa}^{-1}$. The permeability of the LDH/PVA coated layer exhibits more than 26000 fold reduction compared with a pristine PET film and a 266 fold reduction compared with the PVA only coated layer.

Relative permeability is a function of volume fraction $(\varnothing)$ and aspect ratio $(\alpha)$ of inorganic platelet filler.32, 33 Many theoretical models consider the filler shape, array and size distribution. The models considered here ${ }^{1,34-37}$ (a list of the models are included in Table $\mathrm{S}_{5}$, Supporting Information) have the assumption that the platelets are perpendicular to the gas diffusion direction. Both the Nielsen ${ }^{34}$ and the ideal Cussler ${ }^{1}$ model assume the platelets size is uniform and the platelets are regularly arrayed in the polymer matrix. The Cussler-Random array ${ }^{35}$ and Fredrickson-Bicerano ${ }^{37}$ model are based on platelets that have mono-size distribution and the platelets are randomly arrayed. The Gusev-Lusti ${ }^{36}$ model is based on a mathematical approximation from a finite element model that is considered to closely describe the real value of randomly arrayed platelets that has a polysize distribution.

The relative permeability $\left(\mathrm{P} / \mathrm{P}_{\mathrm{o}}\right)$ (Figure 3a, Table $\mathrm{S}_{4}$, Supporting Information) of the LDH/PVA coated layer was plotted as a function of aspect ratio at $48 \mathrm{vol} \% \mathrm{LDH}$ (filler) fraction to compare with theoretical values based on different stacking models ${ }^{1,34-37}$ of an inorganic particle filled polymer composite films. The relative oxygen permeability of the LDH/PVA coated layer decreased as the mean aspect ratio of LDH increased from 87 to 336 whilst the dry coated layer thickness of the film samples remained similar at around $700 \mathrm{~nm}$ (Figure $\mathrm{S}_{11}$ and Table $\mathrm{S}_{4}$ ). The relative oxygen permeability (Figure 3a, Table $\mathrm{S}_{4}$, Supporting Information) is 0.0065 when the LDH sample has an aspect ratio of $87 \pm 17$ and it decreased to 0.0051 when the aspect ratio of LDH sample is increased to $120 \pm 58$ and stayed at 0.0051 at $150 \pm 80$. The relative permea- 

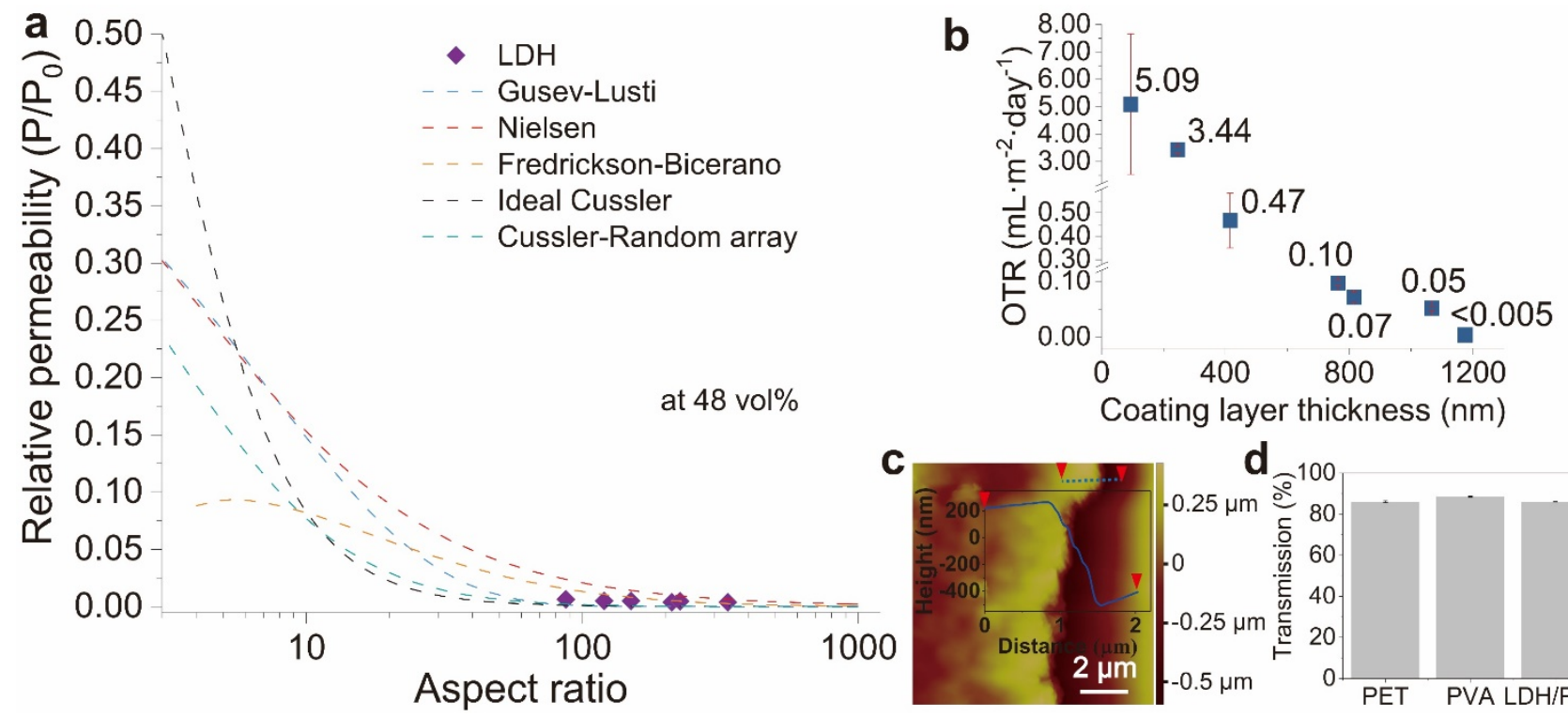

$\begin{array}{llr}400 & 800 & 1200 \\ \text { Coating layer thickness }(\mathrm{nm})\end{array}$

Figure 3. Oxygen barrier performance and physical properties of coated films. a) Theoretical simulations of the relative oxygen permeability of coated films plotted as a function of platelet aspect ratio; experimental data ( $\diamond)$ for films formed using a coating solution containing $3 \mathrm{wt} \% \mathrm{LDH}$ and $2 \mathrm{wt} \%$ PVA using a $24 \mu \mathrm{m}$ gap coating rod. b) OTR as a function of dry coating layer thickness. c) AFM image showing dry coating layer thickness of the film coated with $3 \mathrm{wt} \% \mathrm{LDH}$ and $2 \mathrm{wt} \%$ PVA using a $24 \mu \mathrm{m}$ gap rod. d) Transparency of the PET substrate, $5 \mathrm{wt} \%$ PVA coated PET, and $5 \mathrm{wt} \% \mathrm{LDH} / \mathrm{PVA}$ (3 wt $\%$ base LDH+ $2 \mathrm{wt} \%$ PVA) coated PET films using a $24 \mu \mathrm{m}$ gap rod at wavelength of $550 \mathrm{~nm}$. The LDH sample with aspect ratio of $226 \pm 140$ was selected as a base LDH sample to make coated films in b, c, and d.

bility decreased to 0.0040 when the aspect ratio is $211 \pm 92$ and then increased a very small value to 0.0046 when the aspect ratio is $226 \pm 140$. This unexpected increase can be ascribed to the large coating thickness derivation measured at the film containing LDH with an aspect ratio of 211 \pm 92 . The relative permeability further decreased to 0.0038 when the aspect ratio is $336 \pm 170$ (The OTR value and coated layer thickness are summarized in Table $\mathrm{S}_{4}$, Supporting Information). The relative permeability of the PET film coated using these LDH dispersions in PVA is in good agreement with theoretical predictions using the Fredrickson-Bicerano and Gusev-Lusti models that assume a random array of particles. The dry weight of $\mathrm{LDH}$ nanosheet per $\mathrm{m}^{2}$ coating film is low at ca. 0.24 to $1.2 \mathrm{~g} / \mathrm{m}^{2}$ (Table $\mathrm{S}_{4}$ and Table S6, Supporting Information) compared to most films containing aluminum foil of above $10 \mathrm{~g} / \mathrm{m}^{2} .^{38,39}$

The effect of coated layer thickness on oxygen barrier performance (Figure 3b) was examined. The LDH NS sample $\left(550{ }^{\circ} \mathrm{C}-48 \mathrm{~h}\right.$ ) with a mean aspect ratio of 226 was used as a base $\mathrm{LDH}$ platelet sample to prepare coating solution with a total solid content of $5 \mathrm{wt} \%$. We applied the coating solution on PET substrate using coating rod with coating gap of $6,12,24$, and $40 \mu \mathrm{m}$ to prepare barrier film samples, giving dried coated layer thickness of $94 \pm 12,246 \pm 20,764 \pm$ 52, and $1067 \pm 90 \mathrm{~nm}$ (Figure 3c, Figure S12 and Table S6) respectively. After coating, the barrier film remained as transparent as the PET substrate (Figure 3d). The haze of uncoated PET substrate, PVA coated PET and LDH/PVA coated PET films are 4.9, 3.5, and 9.2 \% respectively (Figure S13). The barrier film with a coating layer thickness of ca. $94 \pm 12 \mathrm{~nm}$ (prepared from a $6 \mu \mathrm{m}$ coating rod) showed an OTR of $5.09 \mathrm{~mL} \cdot \mathrm{m}^{-2} \cdot \mathrm{day}^{-1}$. When using a coating rod with a larger gap $(12 \mu \mathrm{m})$ to prepare the barrier film, the coated layer thickness increase to ca. $246 \pm 20 \mathrm{~nm}$, a two-fold increase. However, the OTR of the coated film showed less than 2 times reduction to $3.44 \mathrm{~mL} \cdot \mathrm{m}^{-2} \cdot \mathrm{day}^{-1}$. Upon increasing the coated layer thickness to ca. $764 \pm 52 \mathrm{~nm}$ (ca. 8 times thicker) (prepared from a $24 \mu \mathrm{m}$ coating rod), the OTR shows ca. 51 times reduction to $0.1 \mathrm{~mL} \cdot \mathrm{m}^{-2} \cdot \mathrm{day}^{-1}$. When the coating layer thickness reached ca. $1067 \pm 90 \mathrm{~nm}$ (ca. 11 times thicker) (prepared from $40 \mu \mathrm{m}$ coating rod), the OTR decreased to a very low value of $0.05 \mathrm{~mL} \cdot \mathrm{m}^{-2}$.day ', showed ca. 100 times reduction.

We also varied the total solid content (LDH and PVA) (of coating solution) to study the coating layer thickness effect on OTR of our barrier films. Using the same base LDH sample, we prepared coating solutions with total solid contents of $3 w t \%(1.8 w t \% \mathrm{LDH}+1.2 w \mathrm{t} \%$ PVA), $7 \mathrm{wt} \%(4.2 w \mathrm{t} \%$ $\mathrm{LDH}+2.8 w \mathrm{t} \%$ PVA), and $10 \mathrm{wt} \%$ (6 wt $\% \mathrm{LDH}+4 \mathrm{wt} \%$ PVA) to coat on PET substrate using a coating rod with 24 $\mu \mathrm{m}$ gap, giving a coating layer thickness of $415 \pm 65,816 \pm$ 59, and $1175 \pm 101 \mathrm{~nm}$ (Figure S14 and Table S6) respectively. The OTR of the coated films decreased exponentially with the increased coating layer thickness by increasing total solid content of coating solution (Figure $3 \mathrm{~b}$ ). The OTR is $0.47 \mathrm{~mL} \cdot \mathrm{m}^{-2} \cdot$ day $^{-1}$ when the coating layer is $415 \pm 65 \mathrm{~nm}(3$ wt $\%$ coating solution). The OTR decreased to 0.1 and 0.07 $\mathrm{mL} \cdot \mathrm{m}^{-2} \cdot$ day $^{-1}$ when the coating layer thickness is increased to $816 \pm 59 \mathrm{~nm}$ ( $7 \mathrm{wt} \%$ coating solution). The OTR of the PET substrate decreased to below the instrument detection limit $\left(<0.005 \mathrm{~mL} \cdot \mathrm{m}^{-2} \cdot \mathrm{day}^{-1}\right)$ when the coating layer thickness reaches $1175 \pm 101 \mathrm{~nm}$ (10 wt $\%$ coating solution) (Figure 3b). 


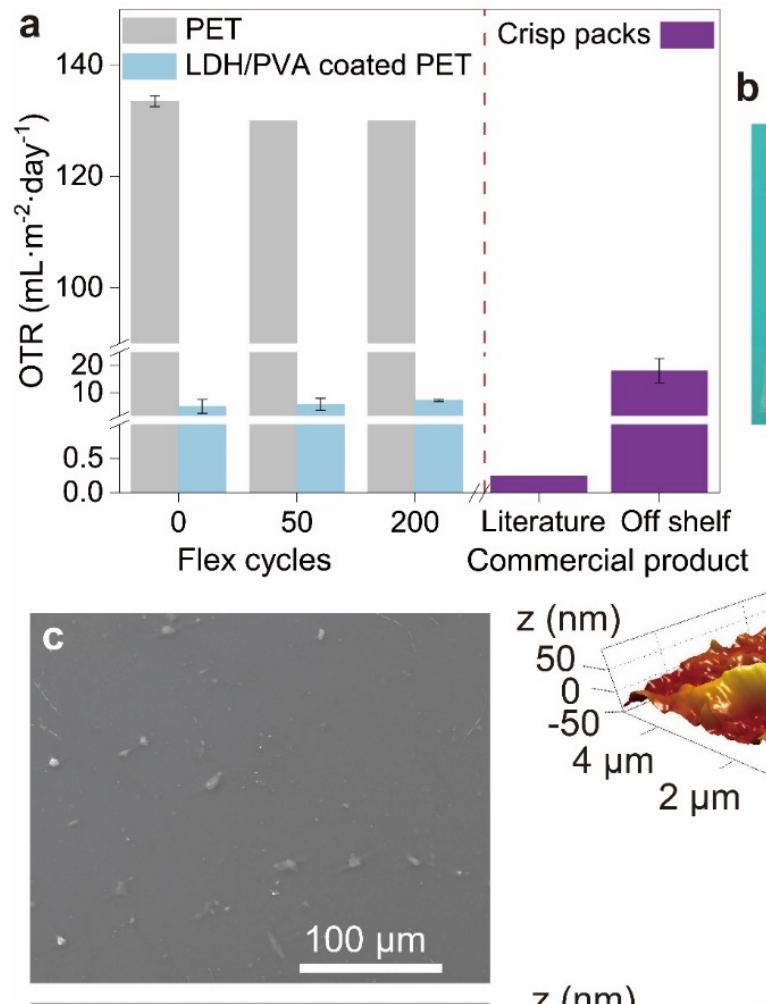

Crisp packs

b Before After 200 flex cycles
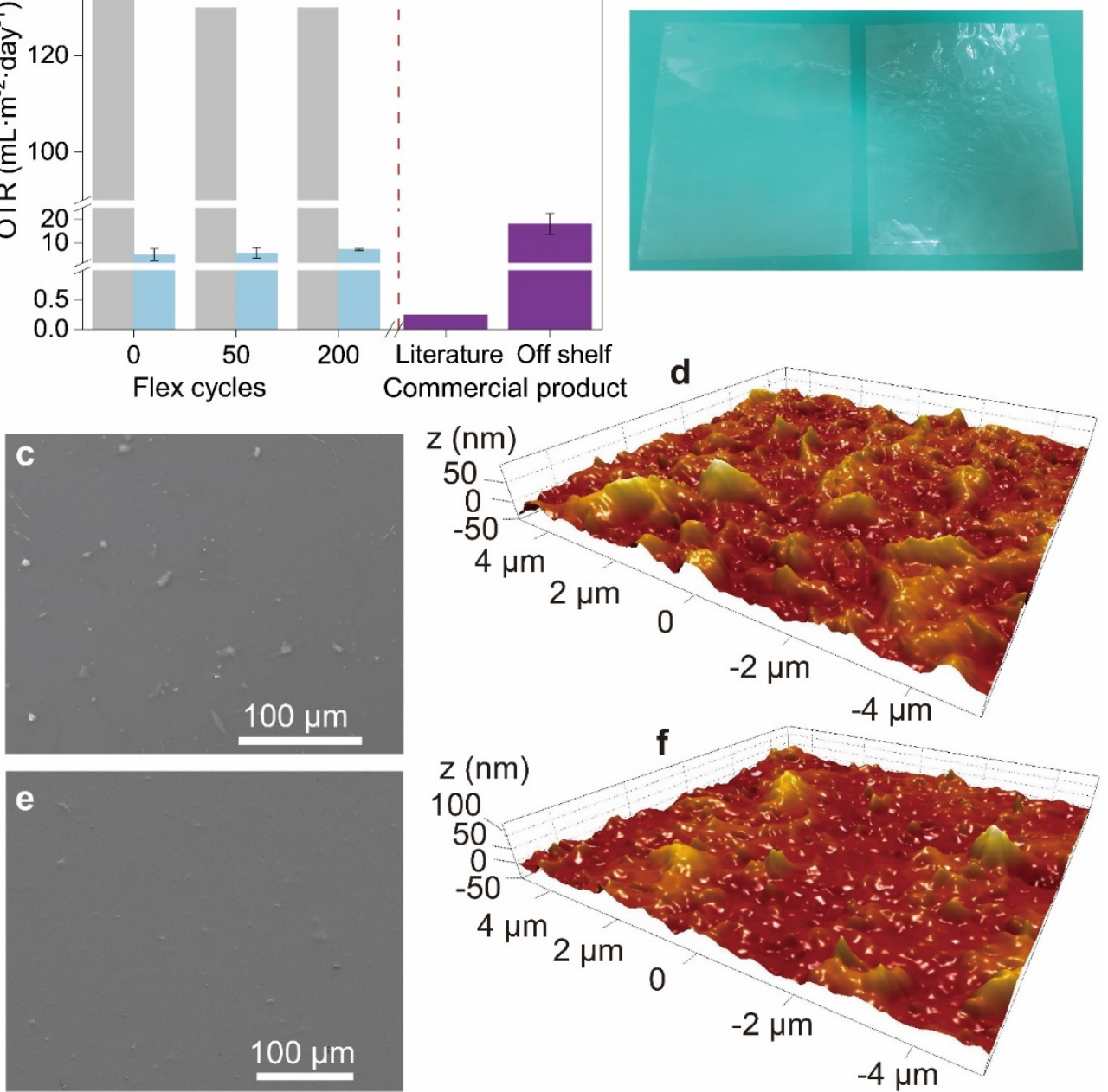

Figure 4. Flexibility of the coated film. a) OTR of the barrier films before, after 50 and 200 flex cycles. The fresh OTR value of a commercial metallized 12 um PET film is from a literature report. $4^{\circ} \mathrm{b}$ ) Digital images of the coated film before and after 200 flex cycles of mechanical flexibility test. SEM image and AFM 3 D topology of the coated film before (c and d) and after 200 cycles of mechanical flexibility test (e and f). The LDH/PVA coated film discussed in this image is prepared by a $3 \mathrm{wt} \% \mathrm{LDH}\left(550{ }^{\circ} \mathrm{C}-48 \mathrm{~h}\right.$ ) and $2 \mathrm{wt} \%$ PVA solution using a $6 \mu \mathrm{m}$ coating rod.

We further tested the OTR and water vapor transmission (WVTR) of the barrier film $(4 \mathrm{wt} \% \mathrm{LDH}+2.8 \mathrm{wt} \% \mathrm{PVA}$ coating solution) at $23{ }^{\circ} \mathrm{C}$ and $60 \%$ relative humidity $(\mathrm{RH})$. The OTR of the film increased from 0.07 to $0.28 \mathrm{~mL} \cdot \mathrm{m}^{-}$ ${ }^{2}$. day $^{-1}$ when the relative humidity increased from o to 60 $\%$ (Figure S15). The WVTR of the PET is decreased from 10 to $2.24 \mathrm{~g} \cdot \mathrm{m}^{-2}$. day $^{-1}$ (Figure $\mathrm{S} 16$ ) when coated with the $4 \mathrm{wt} \%$ $\mathrm{LDH}+\mathbf{2 . 8} \mathrm{wt} \%$ PVA coating solution.

The effectiveness of the barrier coated films was tested by oxygen permeability and barrier improvement factor (BIF) (defined as $P_{s} / P_{t}$, where $P_{s}$ is the permeability of the substrate and $P_{t}$ is the permeability of the coated barrier film). ${ }^{4-43}$ Oxygen permeability quantifies the amount of oxygen gas diffusion through polymer film in unit time per surface area $\left(\mathrm{cm}^{3}\right.$ (STP) $\left.\mathrm{cm} \mathrm{cm}^{-2} \mathrm{~s}^{-1} \mathrm{~Pa}^{-1}\right)$. Permeability includes the thickness of a film that the permeant has to pass through in its calculation. Thus, BIF allows the comparison of oxygen barrier performance across PET barrier films prepared from different technologies. The best performing barrier coated film has a very low permeability of $0.0007 \times$ $10^{-16} \mathrm{~cm}^{3}$ (STP) $\mathrm{cm} \mathrm{cm}^{-2} \mathrm{~s}^{-1} \mathrm{~Pa}^{-1}$ with a BIF of 24319 (Table S6, Supporting Information) which is among the best performing coated films based on PET substrate. In contrast, a commercial metallized $12 \mu \mathrm{m}$ PET film has an oxygen permeability of $0.035 \mathrm{~cm}^{3}$ (STP) $\mathrm{cm} \mathrm{cm}^{-2} \mathrm{~s}^{-1} \mathrm{~Pa}^{-1}$ with a BIF of $678.4^{\circ}$

Pyrolysis is a common method adopted separate the polymer from the thin layer of metal in metallized films. ${ }^{44-46}$ However, the process is energy intensive and results in gasification of the polymers rather recycling of the macromolecule. The bulk PET substrate film layer $(12 \mu \mathrm{m})$ in our oxygen barrier film structure could possibly be recycled by simply washing away the thin LDH/PVA coating layer (ca. $1 \mu \mathrm{m}$ ) in water which should not affect the physical properties of the PET layer.

Mechanical flexibility of LDH nanosheet/PVA coated PET films. During real-life applications, the process of manufacturing, handling and distributing would exert 
large amounts of mechanical stress on food packaging. Whilst commercial metallized films have good gas barrier performance, they are very sensitive to mechanical stress. Typically, a metallized $12 \mu \mathrm{m}$ PET film has an OTR as low as $0.25 \mathrm{~mL} \cdot \mathrm{m}^{-2} \cdot \mathrm{day}^{-1} .^{40}$ The OTR of an off the shelf commercial metallized packaging (with a thickness of ca. $46 \pm 1.1$ $\mu \mathrm{m}$ ) is ca. $18.15 \pm 4.5 \mathrm{~mL} \cdot \mathrm{m}^{-2} \cdot$ day $^{-1}$ (Figure 4a). In contrast, our barrier films are much more resistant to mechanical stress. A Gelbo flex tester was used to simulate the mechanical stresses (twist, pull and compress) and the barrier films were flexed for 50 and 200 cycles. The OTR values (Figure 4a) of both the PET substrate and the LDH/PVA coated PET barrier film remained stable even after 200 flex cycles (Figure $4 \mathrm{~b}$ ). The uncoated PET substrate film exhibited an OTR of $133.5 \pm 1.0 \mathrm{~mL} \cdot \mathrm{m}^{-2} \cdot$ day $^{-1}$ before flexibility tests and exhibited an OTR value of $130.0 \mathrm{~mL} \cdot \mathrm{m}^{-2}$. day ${ }^{-1}$ after 50 as well as 200 flex cycles. The LDH (aspect ratio $=$ 226)/PVA coated PET film exhibited an OTR of $5.09 \pm 2.6$ $\mathrm{mL} \cdot \mathrm{m}^{-2} \cdot \mathrm{day}^{-1}$ before flexibility tests and showed OTR values of $5.8 \pm 2.3$ and $7.23 \pm 0.4 \mathrm{~mL} \cdot \mathrm{m}^{-2}$. day ${ }^{-1}$ after 50 and 200 flex cycles respectively. The results indicated that our barrier films are much more flexible than a metallized layer. The surface morphology of the barrier film before (Figure $4 \mathrm{C}$ and $\mathrm{d}$ ) and after vigorous flex tests (200 cycles) (Figure 4e and f) was further examined by SEM. We did not observe any defects on the coated film subjected to 200 flex cycles which again indicating that the barrier film is flexible and highly resistant to mechanical stresses.

\section{CONCLUSIONS}

In conclusion, non-toxic LDH nanosheet samples with different aspect ratios were prepared and the oxygen barrier performance of the PET films coated with these LDH nanosheets dispersed in PVA was enhanced when the aspect ratio of the LDH nanosheets was the highest. We found that the highest aspect ratio LDH nanosheets were better aligned in the coated layer. This thin layer of nontoxic $\mathrm{Mg}_{2} \mathrm{Al}-\mathrm{LDH} / \mathrm{PVA}$ coated on $12 \mu \mathrm{m}$ PET film exhibit a limiting OTR below instrument detection limit $(<0.005$ $\mathrm{mL} \cdot \mathrm{m}^{-2} \cdot$ day $^{-1}$ ), with good resistance to mechanical stress and high transparency. We expect this new LDH nanosheets dispersion technology to offer new recycling possibilities by eliminating the metallization layer that is the current commercial standard.

\section{METHODS}

Materials: The original platelet $\mathrm{Mg}_{2} \mathrm{Al}-\mathrm{LDH}$ sample and a polyethylene terephthalate (PET) film ( $12 \mu \mathrm{m}$ thick) were used as obtained from SCG Chemicals Co., Ltd. (Thailand). Polyvinyl alcohol (PVA) 8-88 ( $\mathrm{M}_{\mathrm{w}}$ : 67,00o g/mol), glycine $(\geq 98 \%)$, and sodium hydroxide pellets $(\geq 98 \%)$ were purchased from Sigma Aldrich.

Calcination of LDHs: The LDH samples are calcined at 450, 550 , or $750^{\circ} \mathrm{C}$ for $12 \mathrm{~h}$ at a heating rate of $10^{\circ} \mathrm{C} / \mathrm{min}$ in air. The calcined LDOs are removed from the furnace at ca. 8o ${ }^{\circ} \mathrm{C}$ and stored in a desiccator to avoid slow rehydration in air.

Reconstruction of LDOs in amino acid solution (LDH NS): Typically, glycine was mixed with $1 \mathrm{~g}$ calcined LDO at $3: 2$ weight ratio in $10 \mathrm{~mL}$ water and the mixture was placed in an autoclave and reacted at $100{ }^{\circ} \mathrm{C}$ for a specific period $(16$, 24,48 , or $96 \mathrm{~h}$ ) to obtain a semi-transparent gel. The obtained gel was then re-dispersed by stirring in water (usually $100 \mathrm{~mL}$ ) overnight followed by 5 min sonication.

The LDH NS dispersions are very stable in water and so very difficult to collect by centrifugation, even at high $g$ for extended periods. To improve the solid yield, the LDH suspension was intentionally precipitated by adding $\mathrm{NaOH}$ solutions. Typically, to a solution of $100 \mathrm{~mL}$ dispersed gel ca. 4-5 $\mathrm{mL} \mathrm{NaOH}(4 \mathrm{M})$ was added to precipitate the $\mathrm{LDH}$ NS. The LDH NS was then collected by centrifuge at 35954 $\mathrm{g}$ force for 10 minutes and washed three times with D.I. water for further characterization.

After centrifugation, the collected LDH gel was partially dried at $100{ }^{\circ} \mathrm{C}$ in an oven overnight to determine the solid content (the average solid content of three measurements was used in all cases) for coating solution preparation.

Coating solution preparation: PVA solution was prepared by dissolving PVA resin in water at ca. $90^{\circ} \mathrm{C}$ under reflux for an hour. $10 \mathrm{wt} \%$ PVA stock solution was used to prepare the coating solution. Reconstructed LDH gel was mixed and stirred overnight with $10 \mathrm{wt} \%$ PVA solution and water to afford coating solution with differing total solid content and LDH loading. The coating solutions typically contain $95 \mathrm{wt} \%$ water and $5 \mathrm{wt} \%$ solid where $\mathrm{LDH}$ is $3 \mathrm{wt} \%$ and PVA is $2 \mathrm{wt} \%$.

Coating process: PET substrate was coated with the coating solutions by a semiautomatic coater (K control coater, RK PrintCoat instruments Ltd, UK) at a coating speed equivalent to $10.67 \mathrm{~m} / \mathrm{min}$. After coating, the PET films are dried at room temperature for about $\mathrm{i}$ before testing.

OTR testing: The OTR of the barrier films were tested on M8oo1 oxygen permeation analyzer (Systech Instruments, UK) at $23^{\circ} \mathrm{C}$ and zero relative humidity unless specified as $60 \% \mathrm{RH}$. The instrument testing limit is $0.005 \mathrm{~mL} \cdot \mathrm{m}^{-2}$.day ${ }^{1}$. The testing complies with ASTM D-3985.

WVTR testing: The WVTR of the barrier films were tested on a M70o1 water vapor permeation analyzer (Systech Instruments, UK) at $23{ }^{\circ} \mathrm{C}$ and $60 \%$ relative humidity. The testing complies with ASTM standard F-1249.

TEM measurements of LDHs and cross-sectional TEM sample preparation: All TEM images were obtained on a JEOL JEM-210o transmission electron microscope with an accelerating voltage of $200 \mathrm{kV}$. The coated PET films were first embedded into epoxy, and slices of ca. 80-10o nm thickness were cut on a Reichert-Jung Ultracut E ultramicrotome from the embedded epoxy sample. The slices were deposited on 75-mesh copper grids for imaging.

SEM imaging: SEM images were taken on a JEOL JSM6610LV SEM electron microscope with an operating voltage of $5 \mathrm{kV}$ for film samples and $20 \mathrm{Kv}$ for LDH samples. The samples were first coated with ca. $10 \mathrm{~nm}$ gold before imaging.

Optical properties of the films: Transparency of the films were characterized by an Agilent Cary 4000 UV Vis Spectrometer. The transmission was taken at wavelength of 550 
$\mathrm{nm}$. Haze of the films were tested by a Rhopoint ID-L (Rhopoint Instruments Ltd).

AFM measurements: The coating layer thickness and thickness of LDHs were measured by a NanoScope MultiMode atomic force microscope using tapping mode with a silicon tip coated with aluminum with a force constant of $40 \mathrm{~N} / \mathrm{m}$. LDHs samples were diluted into ca. o.o1 $\mathrm{mM}$ and spin coated on freshly cleaved mica wafer for AFM imaging.

Mechanical flex of the films: The films were conditioned at $23 \pm 2{ }^{\circ} \mathrm{C}$ and $50 \pm 5 \% \mathrm{RH}$ for 48 hours before the flex. All films were flexed by a Gelbo flex tester (IDM instruments) following ASTM F392-93 standard.

Pole figure measurements: For Pole figure measurements a Panalytical X'Pert Pro MRD was used. This is equipped with a parabolic mirror providing intense $\mathrm{Cu} \mathrm{K} \mathrm{K}_{\alpha}$ radiation and a Pixcel detector as a point detector with an $8.5 \mathrm{~mm}$ active length. The mirror provides an intense X-ray beam enabling the detection of weak scattering response. This provides each pole figure with a $2 \theta$ range of $1.5^{\circ}$, allowing us to isolate the scattering from the PVA/LDH and $\mathrm{LDH} /$ glycine phase. The samples were mounted on a glass slide using double-sided tape and oriented so that at $\varphi=0^{\circ}$ the coating layer would face the incident beam.

The pole figure measurement consists of a series of $\varphi$ scans (rotation of the sample about the surface normal) made at a number of different $\psi$ angles (sample tilt angle). Each $\varphi$ scan was from o to $360^{\circ}$ with a $2^{\circ}$ step size and a counting time of $0.88 \mathrm{~s}$ per position. A phi scan was made every $2^{\circ}$ from o to $26^{\circ}$ in $\psi$ giving a total collection time per pole figure of 45 minutes.

Gaussian distribution:

$y=y_{0}+\frac{A e^{\frac{-4 \ln (2)\left(x-x_{C}\right)^{2}}{w^{2}}}}{w \sqrt{\frac{\pi}{4 \ln (2)}}}$

Degree of orientation:

$$
\partial=\frac{180-\text { FWHM }}{180} * 100
$$

where FWHM is the full width at half maximum obtained by pole figure measurements.

Ideal laminate theory:

The permeability $\left(\mathrm{P}_{\mathrm{ILT}}\right)$ of coated film is:

$$
P_{I L T}=\left(\frac{\emptyset_{s}}{P_{s}}+\frac{\emptyset_{b}}{P_{b}}\right)^{-1}, \emptyset_{b}=\frac{d_{b}}{d_{b}+d_{s}}, \emptyset_{s}=1-\emptyset_{b}
$$

Where $d_{s}, \emptyset_{s}$, and $P_{s}$ are the thickness, volume fraction, and permeability of the substrate; $d_{b}, \varnothing_{b}$, and $P_{b}$ are the thickness, volume fraction, and permeability of the coated layer.

Barrier improvement factor: Barrier improvement factor (BIF) is defined as Ps/Pt, where Ps is the permeability of the substrate and Pt is the permeability of the coated substrate.

\section{ASSOCIATED CONTENT}

Supporting Information.

The supporting information is available free of charge via the Internet at http://pubs.acs.org.

Figures S1-S16, Tables S1-S6, and additional references.

\section{AUTHOR INFORMATION}

\section{Corresponding Author}

*E-mail: dermot.ohare@chem.ox.ac.uk

\section{ORCID}

Jingfang Yu: oooo-0oo2-1697-4972

Jean-Charles Buffet: oooo-0oo3-2062-9546

Dermot O'Hare: oooo-0oo1-8054-8751

Funding

This work is funded by SCG Chemicals Co., Ltd. (Thailand) (J.Y. and J.-C.B.).

\section{Notes}

The authors declare no competing financial interest.

\section{ACKNOWLEDGMENT}

We thank Dr. Steven Huband (University of Warwick) for pole figure measurements and discussions, Dr. Kanittika Ruengkajorn (SCG Public Packaging Co. Ltd., Bangkok, Thailand) for help with mechanical flex tests of the films, and Research Complex at Harwell (RCaH, Didcot, UK) for instrument time. We thank Dr. Dana Georgiana Crivoi for useful discussions.

\section{REFERENCES}

1. Cussler, E. L.; Hughes, S. E.; Ward, W. J.; Aris, R., Barrier membranes. Journal of Membrane Science 1988, 38 (2), 161-174.

2. Choudalakis, G.; Gotsis, A., Permeability of polymer/clay nanocomposites: a review. Eur. Polym. J. 2oo9, 45 (4), 967-984.

3. Ding, F.; Liu, J.; Zeng, S.; Xia, Y.; Wells, K. M.; Nieh, M.P.; Sun, L., Biomimetic nanocoatings with exceptional mechanical, barrier, and flame-retardant properties from large-scale one-step coassembly. Sci. Adv. 2017, 3 (7), e1701212.

4. Das, P.; Malho, J.-M.; Rahimi, K.; Schacher, F. H.; Wang, B.; Demco, D. E.; Walther, A., Nacre-mimetics with synthetic nanoclays up to ultrahigh aspect ratios. Nat. Commun. 2o15, 6, 5967.

5. $\quad$ Song, Y.; Gerringer, J.; Qin, S.; Grunlan, J. C., High oxygen barrier thin film from aqueous polymer/clay slurry. Industrial $\mathcal{E}$ Engineering Chemistry Research 2018, 57 (20), 6904-6909.

6. Guan, Y.; Meyers, K. P.; Mendon, S. K.; Hao, G.; Douglas, J. R.; Trigwell, S.; Nazarenko, S. I.; Patton, D. L.; Rawlins, J. W., Ecofriendly fabrication of modified graphene oxide latex nanocomposites with high oxygen barrier performance. ACS applied materials $\mathcal{E}$ interfaces 2016, 8 (48), 33210-33220.

7. Liu, H.; Wu, J.; Liu, C.; Pan, B.; Kim, N. H.; Lee, J. H., Differently-charged graphene-based multilayer films by a layer-bylayer approach for oxygen gas barrier application. Composites Part B: Engineering 2018, 155, 391-396.

8. Heo, J.; Choi, M.; Hong, J., Facile Surface Modification of Polyethylene Film via Spray-Assisted Layer-by-Layer Self-Assembly of Graphene Oxide for Oxygen Barrier Properties. Scientific reports 2019, $9(1), 1-7$.

9. Wong, M.; Ishige, R.; White, K. L.; Li, P.; Kim, D.; Krishnamoorti, R.; Gunther, R.; Higuchi, T.; Jinnai, H.; Takahara, A., Large-scale self-assembled zirconium phosphate smectic layers via a simple spray-coating process. Nature communications 2014, 5, 3589.

10. Ra, H. N.; Kim, H. G.; Kim, H.; Peak, S. H.; Kim, Y. C.; Kim, S. S., Effects of size and aspect ratio of zirconium phosphate (ZrP) on barrier properties of epoxy-ZrP nanocomposites. Prog. Org. Coat. 2019, 133, 1-7.

11. Richardson, J. J.; Björnmalm, M.; Caruso, F., Technologydriven layer-by-layer assembly of nanofilms. Science 2015, 348 (6233), aaa2491.

12. Yu, J.; Liu, J.; Clearfield, A.; Sims, J. E.; Speiegle, M. T.; Suib, S. L.; Sun, L., Synthesis of Layered Double Hydroxide SingleLayer Nanosheets in Formamide. Inorg. Chem. 2016. 
13. Dou, Y.; Pan, T.; Xu, S.; Yan, H.; Han, J.; Wei, M.; Evans, D. G.; Duan, X., Transparent, Ultrahigh - Gas - Barrier Films with a Brick - Mortar - Sand Structure. Angew. Chem. Int. Ed. 2015, 54 (33), 9673-9678.

14. Wang, L.; Dou, Y.; Wang, J.; Han, J.; Liu, L.; Wei, M., Layer-by-layer assembly of layered double hydroxide/rubber multilayer films with excellent gas barrier property. Composites Part A: Applied Science and Manufacturing 2017, 102, 314-321.

15. MgAl-Layered Double Hydroxides have EU Approval for Food Contact (PM/REF: 34690 E 60080 , http://www.legislation.gov.uk/uksi/2006/1401/pdfs/uksi 20061401 en. pdf) and US FDA registration as GRAS (UNII: ${ }_{17432 C G 1 K U}$, https://drugs.ncats.io/drug/17432CG1KU).

16. Yu, J.; Wang, Q.; O'Hare, D.; Sun, L., Preparation of two dimensional layered double hydroxide nanosheets and their applications. Chem. Soc. Rev. 2017, 46 (19), 5950-5974.

17. Yu, J.; Martin, B. R.; Clearfield, A.; Luo, Z.; Sun, L., Onestep direct synthesis of layered double hydroxide single-layer nanosheets. Nanoscale 2015, 7 (21), 9448-9451.

18. Hu, G.; Wang, N.; O'Hare, D.; Davis, J., Synthesis of magnesium aluminium layered double hydroxides in reverse microemulsions. J. Mater. Chem. 2007, 17 (21), 2257-2266.

19. Yu, J.; Ruengkajorn, K.; Crivoi, D.-G.; Chen, C.; Buffet, J.C.; O'Hare, D., High gas barrier coating using non-toxic nanosheet dispersions for flexible food packaging film. Nature Communications 2019, 10 (1), 2398.

20. Hibino, T.; Kobayashi, M., Delamination of layered double hydroxides in water. J. Mater. Chem. 2005, 15 (6), 653-656.

21. Jaubertie, C.; Holgado, M.; San Roman, M.; Rives, V., Structural characterization and delamination of lactate-intercalated Zn, Al-layered double hydroxides. Chem. Mater. 2006, 18 (13), 3114-3121. 22. Ren, Q.; Wang, G.; Wu, T.; He, X.; Wang, J.; Yang, J.; Yu, C.; Qiu, J., Calcined MgAl-layered double hydroxide/graphene hybrids for capacitive deionization. Industrial $\mathcal{E}$ Engineering Chemistry Research 2018, 57 (18), 6417-6425.

23. Hobbs, C.; Jaskaniec, S.; McCarthy, E. K.; Downing, C.; Opelt, K.; Güth, K.; Shmeliov, A.; Mourad, M. C.; Mandel, K.; Nicolosi, V., Structural transformation of layered double hydroxides: an in situ TEM analysis. npj 2D Materials and Applications 2018, 2 (1), 1-10.

24. Millange, F.; Walton, R. I.; O'Hare, D., Time-resolved in situ X-ray diffraction study of the liquid-phase reconstruction of $\mathrm{Mg}$ $\mathrm{Al}$-carbonate hydrotalcite-like compounds. J. Mater. Chem. 2ooo, 10 (7), 1713-1720.

25. Kuroda, Y.; Oka, Y.; Yasuda, T.; Koichi, T.; Muramatsu, K.; Wada, H.; Shimojima, A.; Kuroda, K., Precise size control of layered double hydroxide nanoparticles through reconstruction using tripodal ligands. Dalton Transactions 2018, 47 (37), 12884-12892.

26. Nakayama, H.; Wada, N.; Tsuhako, M., Intercalation of amino acids and peptides into $\mathrm{Mg}-\mathrm{Al}$ layered double hydroxide by reconstruction method. Int. J. Pharm. 20o4, 269 (2), 469-478.

27. Kanezaki, E., Direct Observation of a Metastable Solid Phase of $\mathrm{Mg} / \mathrm{Al} / \mathrm{CO}_{3}$-Layered Double Hydroxide by Means of High Temperature in S itu Powder XRD and DTA/TG. Inorg. Chem. 1998, 37 (10), 2588-2590.

28. Wyman, J., The Dielectric Constant of Solutions of Dipolar Ions. Chem. Rev. 1936, 19 (3), 213-239.

29. Roberts, A. P.; Henry, B. M.; Sutton, A. P.; Grovenor, C. R. M.; Briggs, G. A. D.; Miyamoto, T.; Kano, M.; Tsukahara, Y.; Yanaka, M., Gas permeation in silicon-oxide/polymer (SiOx/PET) barrier films: role of the oxide lattice, nano-defects and macro-defects. Journal of Membrane Science 2002, 208 (1), 75-88.

30. Qin, S.; Song, Y.; Floto, M. E.; Grunlan, J. C., Combined high stretchability and gas barrier in hydrogen-bonded multilayer nanobrick wall thin films. ACS applied materials $\mathcal{E}$ interfaces 2017, 9 (9), 7903-7907.

31. Smith, R. J.; Long, C. T.; Grunlan, J. C., Transparent Polyelectrolyte Complex Thin Films with Ultralow Oxygen Transmission Rate. Langmuir 2018, 34 (37), 11086-11091.

32. Sun, L.; Boo, W.-J.; Clearfield, A.; Sue, H.-J.; Pham, H., Barrier properties of model epoxy nanocomposites. Journal of Membrane Science 2008, 318 (1-2), 129-136.

33. Joshi, M.; Adak, B.; Butola, B., Polyurethane nanocomposite based gas barrier films, membranes and coatings: A review on synthesis, characterization and potential applications. Prog. Mater Sci. 2018, 97, 230-282.

34. Nielsen, L. E., Models for the permeability of filled polymer systems. Journal of Macromolecular Science-Chemistry 1967, 1 (5), 929-942.

35. Lape, N. K.; Nuxoll, E. E.; Cussler, E., Polydisperse flakes in barrier films. Journal of Membrane Science 2004, 236 (1-2), 29-37.

36. Gusev, A. A.; Lusti, H. R., Rational design of nanocomposites for barrier applications. Adv. Mater. 20o1, 13 (21), 1641-1643.

37. Fredrickson, G. H.; Bicerano, J., Barrier properties of oriented disk composites. The Journal of chemical physics 1999, 110 (4), 2181-2188.

38. Dutta, A.; Dutta, G., Comparing Optimum Barrier Variables of Aluminium and MPET Foil Based Laminates for Coffee Packaging. Journal of Applied Packaging Research 2016, 8 (3), 5.

39. Deshwal, G. K.; Panjagari, N. R., Review on metal packaging: materials, forms, food applications, safety and recyclability. Journal of Food Science and Technology 2019, 1-16.

40. Jamieson, E.; Windle, A., Structure and oxygen-barrier properties of metallized polymer film. J. Mater. Sci. 1983, 18 (1), 64-8o. 41. Priolo, M. A.; Holder, K. M.; Gamboa, D.; Grunlan, J. C., Influence of clay concentration on the gas barrier of clay-polymer nanobrick wall thin film assemblies. Langmuir 2011, 27 (19), 12106-12114. 42. Chen, G.-G.; Fu, G.-Q.; Wang, X.-J.; Gong, X.-D.; Niu, Y.S.; Peng, F.; Yao, C.-L.; Sun, R.-C., Facile synthesis of high strength hot-water wood extract films with oxygen-barrier performance. Scientific reports 2017, 7 (1), 1-11.

43. Zhang, T.; Yu, Q.; Fang, L.; Wang, J.; Wu, T.; Song, P., AllOrganic Multilayer Coatings for Advanced Poly (lactic acid) Films with High Oxygen Barrier and Excellent Antifogging Properties. ACS Applied Polymer Materials 2019, 1 (12), 3470-3476.

44. $\quad$ Rabnawaz, M.; Wyman, I.; Auras, R.; Cheng, S., A roadmap towards green packaging: The current status and future outlook for polyesters in the packaging industry. Green Chemistry 2017, 19 (20), 4737-4753.

45. $\quad$ Czajczyńska, D.; Anguilano, L.; Ghazal, H.; Krzyżyńska, R.; Reynolds, A. J.; Spencer, N.; Jouhara, H., Potential of pyrolysis processes in the waste management sector. Thermal Science and Engineering Progress 2017, 3, 171-197.

46. Kaiser, K.; Schmid, M.; Schlummer, M., Recycling of polymer-based multilayer packaging: a review. Recycling 2018, 3 (1), 1. 


\section{Table of Contents}

Rational synthesis of non-toxic, high aspect ratio LDH nanosheet dispersions for use in a barrier coating for food packaging films is reported. An oxygen transmission rate (OTR) of $<0.005 \mathrm{~mL} \cdot \mathrm{m}^{-2}$.day ${ }^{-1}$ is observed when a PET substrate is coated with LDH nanosheets dispersed in PVA. Furthermore, the OTR of the coated films remain unchanged, even after 200 cycles of mechanical flex.

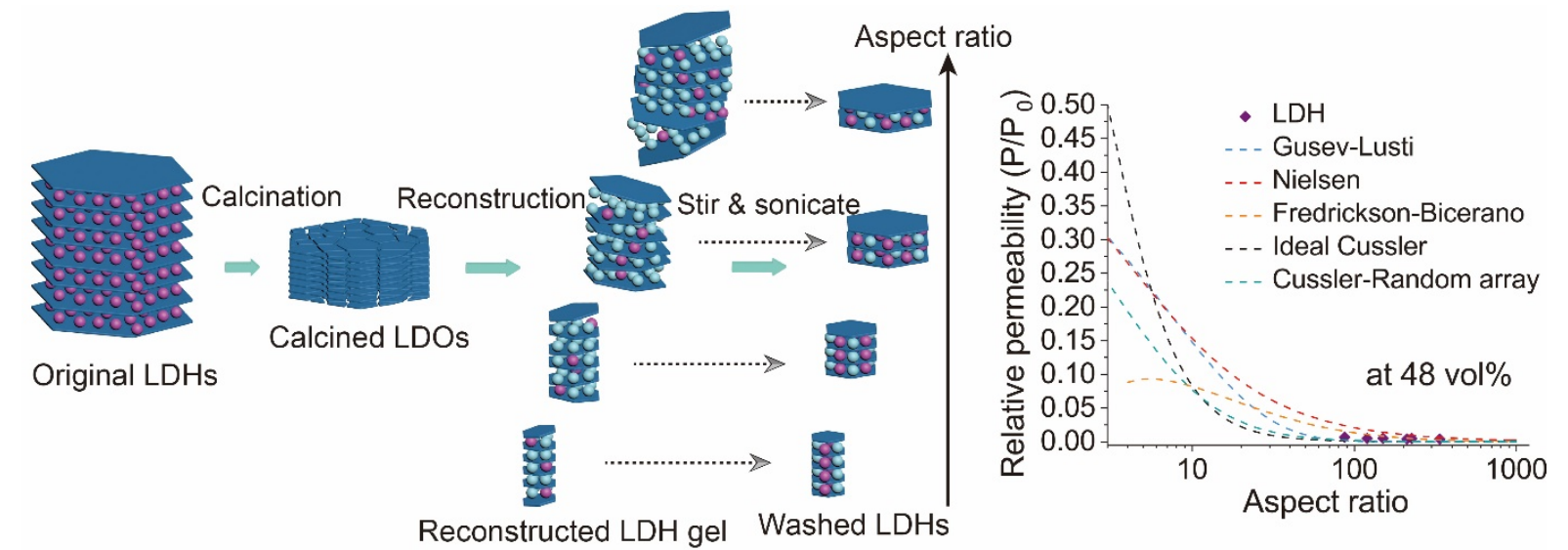

